\title{
Adaptive Transmission Based on MMSE Equalization over Fast Fading Channels
}

\author{
Hongyang Zhang, Xiaojing Huang, J. Andrew Zhang, and Y. Jay Guo \\ University of Technology Sydney, Ultimo, NSW, 2007, Australia \\ Emails: Hongyang. ZHANG-1 estudent . uts.edu.au \\ \{Xiaojing.Huang, Andrew.Zhang, Jay.Guo\}@uts.edu.au
}

\begin{abstract}
The sixth generation (6G) mobile systems will enable high mobility applications in both space and ground based networks. In this paper, we investigate low-complexity equalization and adaptive transmission schemes to combat fast fading channels due to high mobility. We first derive signal and channel models in fast fading channels, which allow low complexity minimum mean square error (MMSE) equalization. We then analyze the output signal-to-noise ratio (SNR) using eigenvalue decomposition for a generalized modulation representation. Assuming the channel state information (CSI) is known at the transmitter, we propose an adaptive transmission technique which utilizes the CSI to precode data symbols in order to improve the output SNR at the receiver. Simulation results show that the adaptive transmission scheme effectively improves the MMSE equalization performance in non-line-of-sight channels especially when the transmission signal frame is short.
\end{abstract}

Index Terms-Adaptive transmission, MMSE equalization, fast fading channel, eigenvalue decomposition

\section{INTRODUCTION}

With the deployment of the fifth generation (5G) mobile networks, the research on next generation technology has become an emerging topic for meeting the demands for higher system capacity and global coverage. The sixth generation (6G) systems will target worldwide low-latency and reliable communications since the ground based communication systems cannot provide fully covered high speed and reliable network to any place on the earth [1]. In particular, the emerging space-air-ground integrated network (SAGIN) combining satellites, aircraft and terrestrial networks will provide seamless connectivity to rural, ocean and mountain areas [2].

For high-mobility situations in space networks, where the wireless channels can be mostly regarded as line-of-sight (LOS) channels, the Doppler frequency shifts caused by relative motion can affect performance significantly. Besides, many ground based communications such as those in vehicular networks, can also work in fast fading non-line-ofsight (NLOS) channels. The 6G technologies should address the high mobility issues under both LOS and NLOS channel conditions.

Conventional modulations, such as orthogonal frequency division multiplexing (OFDM), work well in slow fading channels, since they transmit signals in short frames so that the channel during every frame can be assumed to be constant. However, when the channel coherence time is much smaller than the frame length, it is difficult for conventional modulations to recover signals in such fast fading channels. Although significant efforts on channel estimation, equalization and channel tracking have been made to improve the performance and reduce the pilot overhead, the lack of capability for achieving full time diversity in conventional techniques still limits their applications in high mobility scenarios.

The recently proposed orthogonal time frequency space (OTFS) modulation shows great potential for coping with fast fading channels. Formulating the data in a two dimensional (2D) matrix, OTFS signals can be represented in delayDoppler domain and frequency-time domain [3]. With longer transmission frames, it is easier to equalize signals with multiple Doppler frequency shifts and multipath fading. Current researches on OTFS have been focused on developing more effective and efficient equalization and channel estimation techniques using algorithms like Markov chain Monte Carlo (MCMC), message passing (MP) and so on. However, most of recent studies adopt an effective channel matrix which is a product of permutation matrices and diagonal matrices to incorporate the signal multipath delays and Doppler shifts in their channel models, resulting in complicated channel equalization and signal detection. Some other existing models are developed for OTFS only and cannot be applied to other modulations. Therefore, better general signal and channel models are needed to characterize the fast fading channels, not only for long-frame modulations like OTFS, but also for conventional short-frame modulations such as OFDM and single-carrier frequency-division multiple access (SC-FDMA). Besides, how to improve the performance of conventional short-frame modulations in fast fading channels is of significant importance since a long-frame modulation will inevitably increase the detection complexity and processing delay.

In this paper, we introduce a new channel model and propose an adaptive transmission scheme that allows lowcomplexity minimum mean square error (MMSE) equalization and achieves high output signal-to-noise ratio (SNR) at the receiver. Different from existing OTFS study, we introduce a channel model which demonstrates a circular stripe diagonal characteristic in frequency-Doppler domain. This channel model allows low-complexity MMSE equalization to be used to combat fast fading channels. We then analyze the output SNR of the received signals after MMSE equalization by 
using eigenvalue decomposition. To further improve the output SNR and achieve better performance, we propose an adaptive transmission scheme which uses channel state information (CSI) to precode the data information to be transmitted over the fast fading channels. Simulation results show that our adaptive transmission scheme performs better in NLOS channels, compared with conventional OTFS, SC-FDMA and OFDM.

The rest of the paper is organized as follow. In Section II, channel representation is extended from delay-Doppler domain to different domains over a fast fading channel in continuous and discrete forms respectively. In Section III, the theoretical performance of MMSE equalization is analyzed through eigenvalue decomposition. In Section IV, the adaptive transmission scheme is presented to improve the output SNR at the receiver. Simulation results are shown in Section V and conclusions are drawn in Section VI.

\section{Signal Models}

Considering a single-input single-output (SISO) system and denoting the continuous signal waveform to be transmitted over a fast fading channel as $s(t)$, the received signal can be expressed as

$$
r(t)=\int_{-\infty}^{+\infty} \int_{-\infty}^{+\infty} h(\tau, \nu) s(t-\tau) e^{\mathbf{j} 2 \pi \nu t} d \tau d \nu+w(t)
$$

where $h(\tau, \nu)$ is called the delay-Doppler spreading function of the fast fading channel, $\mathbf{j}=\sqrt{-1}$ and $w(t)$ is the additive white Gaussian noise. For a sparse $P$-path channel, $h(\tau, \nu)$ is defined as

$$
h(\tau, \nu)=\sum_{i=1}^{P} h_{i} \delta\left(\tau-\tau_{i}\right) \delta\left(\nu-\nu_{i}\right)
$$

where $h_{i}, \tau_{i}$, and $\nu_{i}$ are the path gain, delay and Doppler shift of the $i$-th path, respectively, and $\delta(\cdot)$ denotes the Dirac delta function satisfying

$$
\int_{-\infty}^{+\infty} \delta(x) d x=1
$$

Applying Fourier transform (FT) and inverse Fourier transform (IFT) to $h(\tau, \nu)$ with respect to delay $\tau$ and Doppler frequency $\nu$ respectively, the channel's frequency-Doppler domain representation $H_{\nu}(f, \nu)$ and delay-time domain representation $h_{t}(\tau, t)$ can be obtained. In addition, the channel's frequency-time domain representation $H(f, t)$ can be obtained after applying 2D FT with respect to $\tau$ and $\nu$. The relationships among these channel representations in different domains are shown in Fig. 1.

Note that the recent studies on OTFS system in [4], [5] adopt a different received signal model which is expressed as

$$
\begin{aligned}
r(t) & =\int_{-\infty}^{+\infty} \int_{-\infty}^{+\infty} h(\tau, \nu) s(t-\tau) e^{\mathbf{j} 2 \pi \nu(t-\tau)} d \tau d \nu+w(t) \\
& =\int_{-\infty}^{+\infty} \int_{-\infty}^{+\infty} h^{\prime}(\tau, \nu) s(t-\tau) e^{\mathbf{j} 2 \pi \nu t} d \tau d \nu+w(t),
\end{aligned}
$$

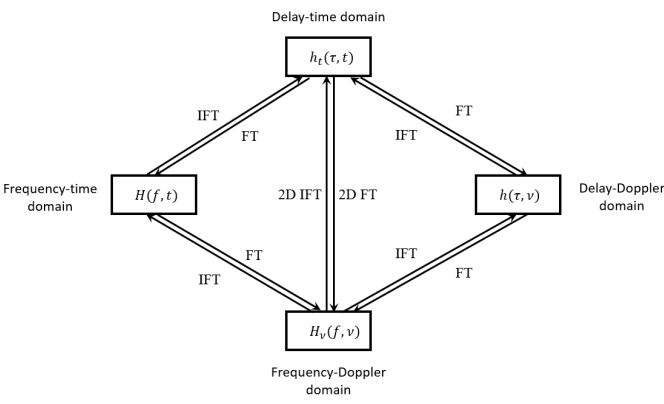

Fig. 1. Relationships among channel representations in different domains.

where $h^{\prime}(\tau, \nu)=h(\tau, \nu) e^{-\mathbf{j} 2 \pi \nu \tau}$. With this model, the relationships shown in Fig.1 and the discrete signal models to be discussed below are no longer valid.

Using the delay-time domain channel representation $h_{t}(\tau, t)$, the received signal can be re-written as

$$
r(t)=\int_{-\infty}^{+\infty} h_{t}(\tau, t) s(t-\tau) d \tau+w(t) .
$$

Letting $\tau^{\prime}=t-\tau$, we have

$$
r(t)=\int_{-\infty}^{+\infty} h_{t}\left(t-\tau^{\prime}, t\right) s\left(\tau^{\prime}\right) d \tau^{\prime}+w(t) .
$$

To derive the received signal model in discrete domain, we first assume that the discrete-time transmitted signal vector $\mathbf{s}=(s[0], s[1], \ldots, s[M N-1])^{T}$ is the vectorized form of an $M$-by- $N$ data symbol matrix with the discrete data symbols $s[i]=s\left(i d_{r}\right), i=0,1, \ldots, M N-1$, where $d_{r}$ is the sampling period, also called the delay resolution. The discrete channel in the delay-time domain can be expressed as

$$
h_{t}[i, j]=h_{t}\left(i d_{r}, j d_{r}\right)=\int_{-1 / 2 d_{r}}^{1 / 2 d_{r}} H\left(f, j d_{r}\right) e^{\mathbf{j} 2 \pi f i d_{r}} d f,
$$

where $H(f, t)$ is derived from (2) as

$$
H(f, t)=\sum_{i=1}^{P} h_{i} e^{-\mathbf{j} 2 \pi f \tau_{i}} e^{\mathbf{j} v 2 \pi \nu_{i} t} .
$$

Therefore, from (6), the discrete time-domain received signal can be expressed in the matrix form as

$$
\mathbf{r}=\mathbf{H}_{t} \mathbf{s}+\mathbf{w},
$$

where $\mathbf{r}$ is the received signal vector,

$$
\mathbf{H}_{t}=\left[\begin{array}{ccc}
h_{t}[0,0] & \cdots & h_{t}[1,0] \\
h_{t}[1,1] & \cdots & h_{t}[2,1] \\
\vdots & \ddots & \vdots \\
h_{t}[M N-1, M N-1] & \cdots & \left.h_{t}[0, M N-1]\right)
\end{array}\right]
$$

denotes the delay-time domain channel matrix and $\mathbf{w}$ is the time-domain noise vector.

Applying discrete Fourier transform (DFT) to (9), we can obtain the discrete frequency-domain received signal as

$$
\mathbf{R}=\mathbf{H}_{\nu} \mathbf{S}+\mathbf{W},
$$


where $\mathbf{R}$ and $\mathbf{S}$ are the transmitted and received signals in the frequency domain respectively,

$$
\begin{aligned}
\mathbf{H}_{\nu} & =\mathbf{F}_{M N} \mathbf{H}_{t} \mathbf{F}_{M N}^{\mathbf{H}} \\
& =\left[\begin{array}{ccc}
H_{\nu}[0,0] & \cdots & H_{\nu}[M N-1,1] \\
H_{\nu}[0,1] & \cdots & H_{\nu}[M N-1,2] \\
\vdots & \ddots & \vdots \\
H_{\nu}[0, M N-1] & \cdots & H_{\nu}[M N-1,0]
\end{array}\right],
\end{aligned}
$$

$\mathbf{F}_{M N}$ and $\mathbf{F}_{M N}^{\mathbf{H}}$ are the DFT and IDFT matrices of length $\mathrm{MN}$ respectively,

$$
H_{\nu}[i, j]=H_{\nu}\left(i f_{r}, j f_{r}\right)=\int_{0}^{1 / f_{r}} H\left(i f_{r}, t\right) e^{-\mathbf{j} 2 \pi j f_{r} t} d t
$$

with $f_{r}$ being the Doppler frequency resolution, and $\mathbf{W}$ is the frequency-Domain noise vector.

The construction of $\mathbf{H}_{t}$ is shown in Fig. 2(a) where the shaded squares stand for the discrete values of $h_{t}[i, j]$ and the blank squares stand for zero elements. The frequencyDoppler domain channel matrix $\mathbf{H}_{\nu}$ demonstrates a circular stripe diagonal property as has been shown in [6] for OFDM systems in time-varying channels. Assuming that the signal frame is long enough to resolve the Doppler frequencies with resolution $f_{r}$, the width of the stripe diagonal is $1+2 K_{D}$ where $K_{D}$ is the Doppler spread. The construction of $\mathbf{H}_{\nu}$ is shown in Fig. 2(b) where the shaded squares stand for the discrete values of $H_{\nu}[i, j]$ and the blank squares stand for zero elements.

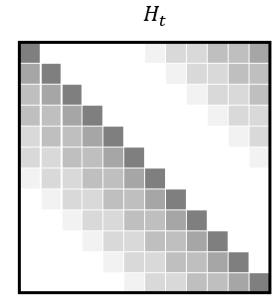

(a)

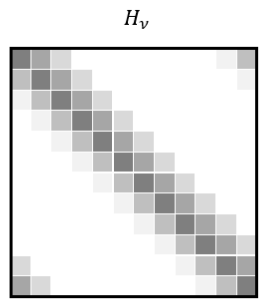

(b)
Fig. 2. Constructions of channel matrices $\mathbf{H}_{t}$ (a) and $\mathbf{H}_{\nu}$ (b) in both time and frequency domain.

Compared with the channel matrix dimension, the width of the diagonal stripe in $\mathbf{H}_{\nu}$ is much smaller for a practical system with moving speed from hundreds to thousands of kilometers per hour, which reduces the cost of matrix inversion significantly and allows for MMSE algorithm to be adopted to equalize the fast fading channels at a low complexity.

\section{OUTPUT SNR ANALYSIS}

Assuming the CSI is already perfectly known at the receiver, MMSE equalization can be used to recover the signals in either time or frequency domain. Due to the equivalence of time and frequency domain equalizations, we only perform frequencydomain analysis. From the frequency domain received signal model in (11), an estimate of the transmitted signal can be expressed as

$$
\hat{\mathbf{S}}=\mathbf{G}_{\nu} \mathbf{R}=\mathbf{G}_{\nu} \mathbf{H}_{\nu} \mathbf{S}+\mathbf{G}_{\nu} \mathbf{W}
$$

where $\mathbf{G}_{\nu}$ is the equalization matrix expressed as

$$
\mathbf{G}_{\nu}=\mathbf{H}_{\nu}^{\mathbf{H}}\left(\mathbf{H}_{\nu} \mathbf{H}_{\nu}^{\mathbf{H}}+\frac{1}{\gamma_{i n}} \mathbf{I}_{M N}\right)^{-1}
$$

$\gamma_{i n}$ denotes the input SNR at the receiver, $\mathbf{I}_{M N}$ denotes the identity matrix of order $M N$ and $(\cdot)^{\mathbf{H}}$ denotes the matrix conjugate and transpose operation of a matrix [7]. Assume that the total number of data symbols to be transmitted is $M \times N$ and let $\mathbf{x}=(x[0], x[1], \ldots, x[M N-1])^{T}$ denote the data symbol vector after the quadrature amplitude modulation (QAM) symbol mapping. Let $\mathbf{S}=\mathbf{F}_{M N} \mathbf{V x}$ where $\mathbf{V}$ and $\mathbf{V}^{\mathbf{H}}$ denote the modulation and demodulation matrices, respectively, satisfying $\mathbf{V} \mathbf{V}^{\mathbf{H}}=\mathbf{V}^{\mathbf{H}} \mathbf{V}=\mathbf{I}_{M N}$. A general representation of the received signals after MMSE equalization is given by

$$
\begin{aligned}
\mathbf{y} & =\mathbf{V}^{\mathbf{H}} \mathbf{F}_{M N}^{\mathbf{H}} \hat{\mathbf{S}} \\
& =\mathbf{V}^{\mathbf{H}} \mathbf{F}_{M N}^{\mathbf{H}} \mathbf{G}_{\nu} \mathbf{H}_{\nu} \mathbf{F}_{M N} \mathbf{V} \mathbf{x}+\mathbf{V}^{\mathbf{H}} \mathbf{F}_{M N}^{\mathbf{H}} \mathbf{G}_{\nu} \mathbf{W} .
\end{aligned}
$$

Different modulation schemes can be realized by selecting different $\mathbf{V}$ matrices. To be specific, $\mathbf{V}=\mathbf{F}_{M}^{\mathbf{H}}$ represents OFDM, $\mathbf{V}=\mathbf{I}_{M}$ represents SC-FDMA and $\mathbf{V}=\mathbf{F}_{N}^{\mathbf{H}} \otimes \mathbf{I}_{M}$ represents OTFS. Note that for different modulations, the dimension of channel matrix and frame length may be different.

Defining $\mathbf{A}=\mathbf{V}^{\mathbf{H}} \mathbf{F}_{M N}^{\mathbf{H}} \mathbf{G}_{\nu} \mathbf{H}_{\nu} \mathbf{F}_{M N} \mathbf{V}$, which represents the signal transformation from $\mathbf{x}$ to $\mathbf{y}$ in (16), and using (15), we can obtain

$$
\begin{aligned}
\mathbf{A} & =\mathbf{V}^{\mathbf{H}} \mathbf{F}_{M N}^{\mathbf{H}} \mathbf{H}_{\nu}^{\mathbf{H}}\left(\mathbf{H}_{\nu} \mathbf{H}_{\nu}^{\mathbf{H}}+\frac{1}{\gamma_{i n}} \mathbf{I}\right)^{-1} \mathbf{H}_{\nu} \mathbf{F}_{M N} \mathbf{V} \\
& =\mathbf{V}^{\mathbf{H}} \mathbf{F}_{M N}^{\mathbf{H}}\left(\mathbf{I}+\frac{1}{\gamma_{i n}}\left(\mathbf{H}_{\nu}^{\mathbf{H}} \mathbf{H}_{\nu}\right)^{-1}\right)^{-1} \mathbf{F}_{M N} \mathbf{V} \\
& =\mathbf{V}^{\mathbf{H}}\left(\mathbf{I}+\frac{1}{\gamma_{i n}}\left(\mathbf{F}_{M N}^{\mathbf{H}} \mathbf{H}_{\nu}^{\mathbf{H}} \mathbf{H}_{\nu} \mathbf{F}_{M N}\right)^{-1}\right)^{-1} \mathbf{V} \\
& =\left(\mathbf{I}+\frac{1}{\gamma_{i n}}\left(\mathbf{V}^{\mathbf{H}} \mathbf{F}_{M N}^{\mathbf{H}} \mathbf{H}_{\nu}^{\mathbf{H}} \mathbf{H}_{\nu} \mathbf{F}_{M N} \mathbf{V}\right)^{-1}\right)^{-1} .
\end{aligned}
$$

Note that $\mathbf{H}_{\nu}^{\mathbf{H}} \mathbf{H}_{\nu}$ is a Hermitian matrix so that it can be expressed using eigenvalue decomposition as $\mathbf{H}_{\nu}^{\mathbf{H}} \mathbf{H}_{\nu}=$ $\mathbf{Q} \Lambda \mathbf{Q}^{\mathbf{H}}$, where $\mathbf{Q}$ is a square $M N \times M N$ unitary matrix and $\boldsymbol{\Lambda}$ is a diagonal matrix with the $i$-th diagonal element $\lambda_{i}$ for $i=0,1, \ldots, M N-1$. Further denoting $\mathbf{U}=\mathbf{V}^{\mathbf{H}} \mathbf{F}_{M N}^{\mathbf{H}} \mathbf{Q}$, we can simplify $\mathbf{A}$ as

$$
\begin{aligned}
\mathbf{A} & =\left(\mathbf{I}+\frac{1}{\gamma_{i n}} \mathbf{U} \boldsymbol{\Lambda}^{-\mathbf{1}} \mathbf{U}^{\mathbf{H}}\right)^{-1} \\
& =\mathbf{U}\left(\mathbf{I}+\frac{1}{\gamma_{i n}} \boldsymbol{\Lambda}^{-\mathbf{1}}\right)^{-1} \mathbf{U}^{\mathbf{H}} \\
& =\mathbf{U}\left(\operatorname{diag}\left(1+\frac{1}{\gamma_{i n} \lambda_{i}}\right)\right)^{-1} \mathbf{U}^{\mathbf{H}} \\
& =\mathbf{U d i a g}\left(\frac{\gamma_{i n} \lambda_{i}}{\gamma_{i n} \lambda_{i}+1}\right) \mathbf{U}^{\mathbf{H}} \\
& =\mathbf{I}-\mathbf{U d i a g}\left(\frac{1}{\gamma_{i n} \lambda_{i}+1}\right) \mathbf{U}^{\mathbf{H}},
\end{aligned}
$$


where $\operatorname{diag}\left(x_{i}\right)$ denotes a diagonal matrix with the $i$-th diagonal element $x_{i}$. According to the MMSE equalization principle, the normalized noise power for the $(n M+m)$-th equalized data symbol, $m=0,1, \ldots, M-1$ and $n=0,1, \ldots, N-1$, can be expressed as

$$
\begin{aligned}
J_{n M+m} & =1-\mathbf{A}[n M+m, n M+m] \\
& =\sum_{i=0}^{M N-1} \frac{1}{\gamma_{i n} \lambda_{i}+1}|\mathbf{U}[n M+m, i]|^{2} .
\end{aligned}
$$

Therefore, the output SNR for the $(n M+m)$-th data symbol after equalization can be expressed as

$$
\begin{aligned}
\gamma_{\text {out }}[m, n] & =\frac{1-J_{n M+m}}{J_{n M+m}} \\
& =\frac{1}{J_{n M+m}}-1 .
\end{aligned}
$$

\section{ADAPTIVE TRANSMISSION}

Assuming that the CSI can be fed-back to the transmitter, we can minimize the normalized noise power (19) by precoding the transmitted data symbols based on the the channel conditions, resulting in an adaptive transmission system. This can be achieved by constructing a modulation matrix $\mathbf{V}$ such that $\mathbf{U}$ satisfies

$$
|\mathbf{U}[n M+m, i]|^{2}=\frac{1}{M N},
$$

and the optimized output SNR becomes

$$
\gamma_{\text {out }}^{*}[m, n]=\frac{1}{\frac{1}{M N} \sum_{i=0}^{M N-1} \frac{1}{\gamma_{i n} \lambda_{i}+1}}-1 .
$$
have

Letting $\mathbf{V}=\mathbf{F}_{M N}^{\mathbf{H}} \mathbf{Q F} \mathbf{F}_{M N}$ and substituting it into $\mathbf{U}$, we

$$
\mathbf{U}=\mathbf{F}_{M N}^{\mathbf{H}} \mathbf{Q}^{\mathbf{H}} \mathbf{F}_{M N} \mathbf{F}_{M N}^{\mathbf{H}} \mathbf{Q}=\mathbf{F}_{M N}^{\mathbf{H}},
$$

and hence the condition (21) is satisfied. Therefore, the adaptive transmission system can be designed as shown in Fig. 3.

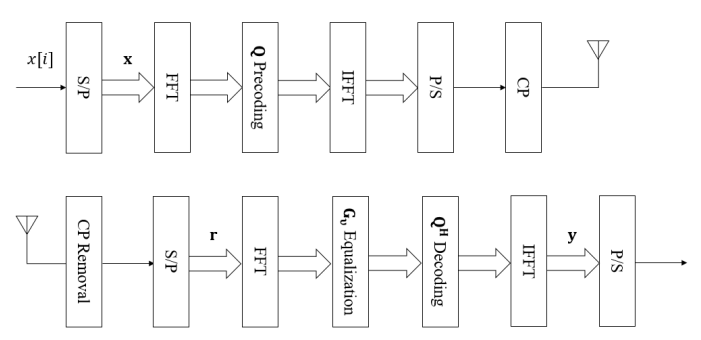

Fig. 3. Adaptive transmission system block diagram: transmitter (upper) and receiver (lower)

At the transmitter of adaptive transmission, the data symbol vector $\mathbf{x}$ is transformed into the frequency domain first and then precoded by the eigenvalue matrix $\mathbf{Q}$. After transforming it back to time domain and appending the cyclic prefix (CP) to the data symbol vector, the data symbol frame is sent over the fast fading channel. At the receiver, after the $\mathrm{CP}$ removal and channel estimation, the CSI is fed-back to the transmitter and the received signal is recovered by frequency-domain MMSE equalization.

Assuming QAM modulation for data symbols, the average bit error rate (BER) for a given channel realization is

$$
P_{b}=\frac{\sum_{m=0}^{M-1} \sum_{n=0}^{N-1} \frac{2\left(1-2^{-k}\right)}{k} Q\left(\sqrt{\frac{3}{4^{k}-1} \gamma_{\text {out }}[m, n]}\right)}{M N},
$$

where the Q-function is defined as

$$
Q(x)=\frac{1}{\sqrt{2 \pi}} \int_{x}^{\infty} e^{-\frac{t^{2}}{2}} d t,
$$

and $2^{2 k}$ indicates the modulation level. For example, $k=1$ means 4-QAM or QPSK. Averaging over all possible fading channel realizations, the ergodic BER for the fast fading channel is expressed as $E_{h}\left\{P_{b}\right\}$, where $E_{h}\{\cdot\}$ denotes the ensemble average over all delay-Doppler channel realizations.

\section{Simulation}

In this section, BER performance of various modulation schemes using MMSE equalization is compared over fast fading channels. The ETSI tapped delay line (TDL) models, which define the time delay in each fading path depending on the LOS or NLOS channel condition, are adopted to generate multipath channel coefficients [8]. To simulate fast fading channels, Doppler frequency shifts are added to every path obeying uniform distribution within the Doppler frequency range $\left[-K_{D}, K_{D}\right]$. The simulation parameters are listed in Table 1. Different modulation schemes such as OTFS, SC-FDMA, OFDM with different frame lengths and their associated adaptive transmission schemes are simulated and compared. To be specific, there are $M \times N$ data symbols in one OTFS frame and $M$ data symbols in one SC-FDMA or OFDM frame. The two adaptive transmission schemes with frame lengths $M$ and $M N$ are named Adaptive-short and Adaptive-long respectively in the following discussions.

TABLE I

SiMULATION PARAMETERS

\begin{tabular}{|c|c|}
\hline Carrier Frequency & $6 \mathrm{GHz}$ \\
\hline No. of Subcarriers $(M)$ & 256 \\
\hline No. of OFDM/SC-FDMA Symbols $(N)$ & 32 \\
\hline Subcarrier Spacing & $30 \mathrm{KHz}$ \\
\hline Maximum Speed & $500 \mathrm{Km} / \mathrm{h}$ \\
\hline No. of Doppler Shifts (Positive or Negative) $\left(K_{D}\right)$ & $\approx 3$ \\
\hline
\end{tabular}

We firstly assume that the CSI is perfectly known at both receiver and transmitter. Fig. 4 shows the simulated BER performance of OTFS, OFDM, SC-FDMA and their associated adaptive transmission schemes in the fast fading TDL-D LOS channel. We observe that the performance of Adaptive-long is very similar to that of OTFS and better than other modulations, 
achieving $10^{-7}$ BER at $17 \mathrm{~dB}$ SNR. SC-FDMA and Adaptiveshort have about $2 \mathrm{~dB}$ degradation while OFDM is the worst among these modulations.

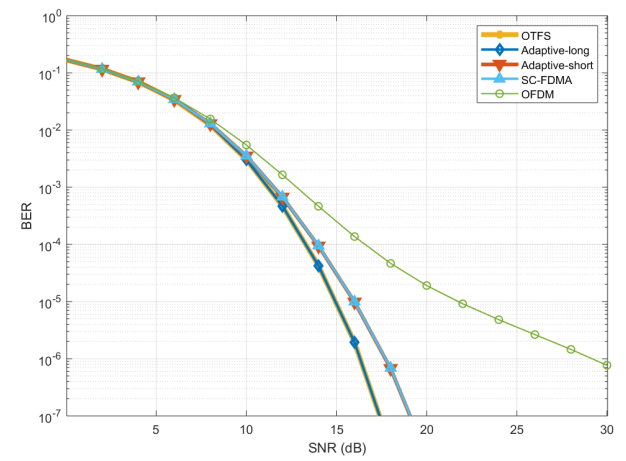

Fig. 4. Comparison of various modulation schemes in LOS channel.

Fig. 5 shows the BER results in fast fading TDL-A NLOS channel. We observe that Adaptive-long demonstrates the best performance, achieving $10^{-7}$ BER at $24 \mathrm{~dB}$ SNR. The performance of OTFS is degraded about $5 \mathrm{~dB}$ at the same BER. The performance of Adaptive-short is slightly degraded as compared to that of OTFS but is much better than that of SC-FDMA. OFDM still shows the worst performance. Since perfect CSI can not be achieved in practice, we also simulate the performance when imperfect channel estimation is applied to the system. Adding a random error matrix obeying Gaussian distribution into the estimated channel matrix, the eigenvector matrix is re-produced for adaptive transmission and equalization. The variance of the channel error matrix is assume to be inversely proportional to the SNR. OTFSpractical and Adaptive-long-practical in Fig. 5 demonstrate the practical performance with channel estimation error. It is shown that the impact of channel estimation error on the performance is significant at lower SNR but tends to be minor at higher SNR. Meanwhile, adaptive transmission still shows better performance than that of OTFS.

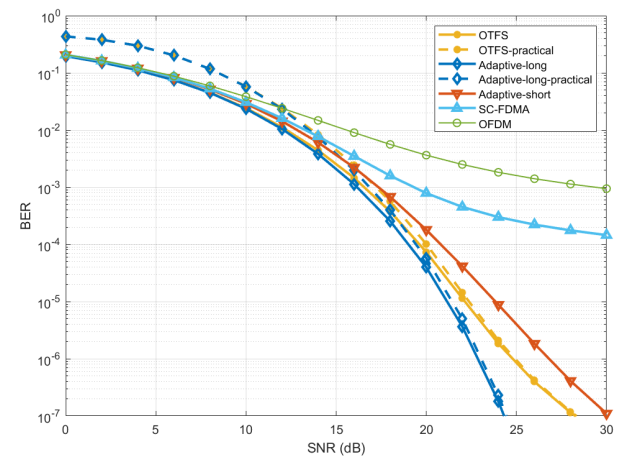

Fig. 5. Comparison of various modulation schemes in NLOS channel.

We see that the adaptive transmission utilizes the eigenvector matrix to precode the data symbols in the frequency domain and hence can alleviate the fast fading effects especially in NLOS channels with low-complexity MMSE equalization. On the other hand, the ability of a modulation scheme to explore frequency diversity and the length of the signal frame also have impacts on system performance. The results show that the long-frame OTFS always outperforms the short-frame SCFDMA and OFDM. The reason is that the long-frame OTFS can resolve the Doppler frequency and achieve full diversity in both time and frequency domain, whereas SC-FDMA can only partially resolve the Doppler frequency and hence has degraded performance. OFDM always performs worst since it only achieves partial time diversity and no frequency diversity.

\section{CONCLUSION}

In this paper, we have derived new signal models and presented an adaptive transmission scheme allowing lowcomplexity MMSE equalization for wireless communication in fast fading channel. The characteristics of frequencyDoppler domain channel matrix makes it possible to adopt low-complexity MMSE equalization. Through channel matrix eigenvalue decomposition, we have proposed an adaptive transmission scheme to improve the equalized signal output SNR. Simulation results show that the proposed adaptive transmission scheme performs well in NLOS channels especially with short signal frames. With the aid of CSI feedback, high performance and low complexity signal equalization with low processing delay can be achieved by the proposed adaptive transmission scheme over fast fading channels. The effectiveness of the proposed adaptive transmission principle is also demonstrated in the cases with practical channel estimation and feedback.

\section{REFERENCES}

[1] X. Huang et al., "Airplane-aided integrated networking for $6 \mathrm{~g}$ wireless: Will it work?" IEEE Vehicular Technology Magazine, vol. 14, no. 3, pp. 84-91, Sep. 2019.

[2] J. Liu, Y. Shi, Z. M. Fadlullah, and N. Kato, "Space-air-ground integrated network: A survey," IEEE Communications Surveys Tutorials, vol. 20 no. 4, pp. 2714-2741, Fourthquarter 2018.

[3] R. Hadani et al., "Orthogonal time frequency space modulation," in 2017 IEEE Wireless Communications and Networking Conference (WCNC), March 2017, pp. 1-6.

[4] P. Raviteja, K. T. Phan, Y. Hong, and E. Viterbo, "Interference cancellation and iterative detection for orthogonal time frequency space modulation," IEEE Transactions on Wireless Communications, vol. 17, no. 10 , pp. 6501-6515, Oct 2018.

[5] S. Tiwari, S. S. Das, and V. Rangamgari, "Low complexity LMMSE receiver for OTFS," IEEE Communications Letters, 2019, Early Access.

[6] F. Hlawatsch and G. Matz, Wireless Communications Over Rapidly TimeVarying Channels, 1st ed. Orlando, FL, USA: Academic Press, Inc., 2011.

[7] X. Huang, "Diversity performance of precoded OFDM with MMSE equalization," in 2007 International Symposium on Communications and Information Technologies, Oct 2007, pp. 802-807.

[8] ETSI, "Study on channel model for frequencies from 0.5 to $100 \mathrm{GHz}$," ETSI TR 138901 V15.0.0, July 2018. 